\title{
Memory and chaos in an Ising spin glass
}

\author{
R. Mathieu, P. E. Jönsson, and P. Nordblad \\ Department of Materials Science, Uppsala University, Box 534, SE-751 21 Uppsala, Sweden \\ H. Aruga Katori and A. Ito \\ RIKEN (The Institute of Physical and Chemical Research), Wako, Saitama, 351-0198, Japan
}

(October 26, 2018)

\begin{abstract}
The non-equilibrium dynamics of the model 3d-Ising spin glass - $\mathrm{Fe}_{0.55} \mathrm{Mn}_{0.45} \mathrm{TiO}_{3}$ - has been investigated from the temperature and time dependence of the zero field cooled magnetization recorded under certain thermal protocols. The results manifest chaos, rejuvenation and memory features of the equilibrating spin configuration that are very similar to those observed in corresponding studies of the archetypal RKKY spin glass $\mathrm{Ag}(\mathrm{Mn})$. The sample is rapidly cooled in zero magnetic field, and the magnetization recorded on re-heating. When a stop at constant temperature $T_{s}$ is made during the cooling, the system evolves toward its equilibrium state at this temperature. The equilibrated state established during the stop becomes frozen in on further cooling and is retrieved on re-heating. The memory of the aging at $T_{s}$ is not affected by a second stop at a lower temperature $T_{s}^{\prime}$. Reciprocally, the first equilibration at $T_{s}$ has no influence on the relaxation at $T_{s}^{\prime}$, as expected within the droplet model for domain growth in a chaotic landscape.
\end{abstract}

\section{INTRODUCTION}

In the droplet model of spin glasses (SG) 1 , the equilibrium dynamics is governed by low energy excitations from the ground state (droplets). If a weak magnetic field - weak enough not to affect the system - is applied, the equilibrium system is magnetized via polarization of droplets of ever increasing size. However, the droplet model SG phase is chaotic 1 in the sense that the equilibrium configuration is unstable to any temperature change $\Delta T$ (or change in the distribution of the interaction) and thus has to rearrange after such a perturbation. For example, after a quench from the paramagnetic phase into the spin glass phase the system is trapped in a random non-equilibrium spin configuration which slowly equilibrates (ages). The aging occurs via droplet excitations which equilibrate the spin configuration on larger and larger length scales, causing the spin glass correlation length to grow. Also, the equilibrium configurations at two different temperatures $T$ and $T+\Delta T$ are only different on large length scales, outside an overlap region. It is observed experimentally that if an equilibrium SG configuration is imprinted up to a certain length scales (age) by keeping the spin glass a certain time at constant temperature, this length scale is conserved even though new configurations on shorter length scales are imprinted at other (lower) temperatures. Manifestations of memory and chaos in spin glasses have been observed in the relaxation and temperature dependence of the low frequency ac-susceptibility in thermal protocols containing halts at constant temperature 3 , as well as in temperature cycling experiments. In addition, signatures of memory and chaos have been demonstrated in simple dc-magnetization vs. temperature measurements 6 on a standard RKKY spin glass, $\mathrm{Ag}(\mathrm{Mn})$. In the present report the model $3 \mathrm{~d}$ Ising spin-glass, $\mathrm{Fe}_{0.55} \mathrm{Mn}_{0.45} \mathrm{TiO}_{3}$, is investigated using dc-magnetization experiments. The temperature dependence, as well as the relaxation of the zero field cooled (ZFC) magnetization is recorded after specific cooling protocols. The results evidence memory and chaos phenomena in agreement with the predictions from the droplet model for the chaotic SG phase.

\section{EXPERIMENTAL}

The ZFC magnetization of a single crystal of $\mathrm{Fe}_{0.55} \mathrm{Mn}_{0.45} \mathrm{TiO}_{3}$ was recorded versus temperature and time in a non-commercial low-field SQUID magnetometert. This spin glass is considered as a canonical short-range Ising spin-glas\$ $\mathbf{Q}$, with a SG transition temperature $T_{g} \sim 22 \mathrm{~K}$. In the experiments, the cooling of the sample was interrupted by stop(s) at constant temperature. In the case of single stop experiments (SSE), the sample was first rapidly cooled in zero magnetic field from a reference temperature $T_{\text {ref }}>T_{g}$ down to the stop temperature $T_{s}<T_{g}$, where it was kept for $t_{s}=3000 \mathrm{~s}$ (and other durations). The cooling was then resumed down to the lowest temperature where the magnetic field $h=1$ Oe was applied, and the magnetization recorded on re-heating. In the double stops experiments (DSE) case, an equally long second temperature stop was made at $T_{s}^{\prime}$ $<T_{s}$. A reference curve was recorded after direct cooling of the sample to the lowest temperature.

The same cooling protocols were employed in the relaxation measurements. After re-heating to the measurement temperature $T_{m}$, and a wait time of $t_{w}=10 \mathrm{~s}$, a magnetic field $\Delta h=2$ Oe was applied, and the magnetization 
recorded vs. time elapsed after the field application. In the SSE protocol, a 3000s stop was made a $T_{s}=T_{m}$ - the experiment is thus comparable to a short negative temperature cycling - while in the DSE, two 3000s stops were made at $T_{s}$ and $T_{s}^{\prime}<T_{s}$, and the relaxation measured at both $T_{m}=T_{s}$ and $T_{m}=T_{s}^{\prime}$. Reference measurements (RE) were performed at $T_{m}$ by cooling the sample directly to the lowest temperature, and recording $M$ vs. time in $\Delta h=2$ Oe after re-heating the sample to $T_{m}$ and employing the wait time of $t_{w}=10 \mathrm{~s}$. "Conventional" ZFC relaxation measurements, in which the sample was only rapidly cooled to $T_{m}$, with $t_{w}=3000 \mathrm{~s}$, were performed for comparison and to ensure that the magnifide of the field was low enough to give a linear response from the system.

The dc magnetic field was applied along the $c$-axis of the single-crystal. It is generated by a small superconductive solenoid coil working in persistent mode during measurements. The time constant of the superconducting magnet is of order $1 \mathrm{~ms}$, but the whole procedure to change the field from $h$ to $h+\Delta h$ and re-establish persistent mode in the magnet takes $\sim 0.2 \mathrm{~s}$ which also determines the shortest observation time in the relaxation experiments. Apart from the stops at constant temperature, the cooling rate was $\sim 4 \mathrm{~K} / \mathrm{min}$, and the heating rate of the order of $1 \mathrm{~K} / \mathrm{min}$.

\section{RESULTS AND DISCUSSION}

Figure 1 illustrates the effects on the ZFC magnetization of a stop at constant temperature during the cooling. As seen in Fig. 1](a), the curve corresponding to the SSE experiments lies significantly below the reference one. This shows that when the system is left unperturbed at constant temperature $T_{s}$ it rearranges its spin configuration toward the equilibrium one for this temperature. The equilibrated state becomes frozen in on further cooling, and is retrieved on re-heating. In other terms, the system remembers its age or shows a memory phenomenon. It should be mentioned that the weak magnetic field only acts as a weak perturbation and has basically no influence on the intrinsic equilibration (aging) of the spin configuration at $T_{s}$. Similar effects would be obtained for example with an ac-field $\mathbf{B}^{\mathbf{3}}$ (as in conventional memory experiments) or when keeping a non-zero magnetic field when performing the stop 10 . This will also be evidenced in the relaxation measurements described below. Fig. 1 (b) presents the difference curves obtained by subtracting from the reference the SSE curves obtained for different stop times $t_{s}$. The stop times are chosen logarithmically spaced, and one immediately observes that the minimum of the different curves are closely equidistant from each other in magnitude, indicating logarithmic relaxation. The advantage of the dc-method to expose fundamental SG features in this simple may has also been demonstrated for other glassy systems.
The effects of two stops during cooling are now studied. Fig. 2 shows the results of DSE at $T_{s 1}=21 \mathrm{~K}$ and $T_{s 2}=17 \mathrm{~K}$. The main frame shows the difference plots for the DSE, as well as the corresponding SSE. The inset depicts the original ZFC magnetization curves before subtraction from the reference. Comparing the DSE curve with the corresponding SSE ones in the main frame, it is clear that the relaxation at $T_{s 1}=21 \mathrm{~K}$ is not affected by the second stop at lower temperature and reciprocally, the aging at $T_{s 2}$ is not influenced by the first equilibration at the higher temperature. This illustrates again the memory and temperature chaos features. During the first stop, the system reaches a characteristic age - or length scale. This age is conserved on further cooling, even if the system is again aged at lower temperature toward its equilibrium state at this temperature12. The state equilibrated at the higher temperature thus survives the spin re-configuration occurring at lower temperature on shorter length scales, and the system remembers its initial high temperature state on re-heating. Of course, if the stop at lower temperature is performed for a much longer time, allowing the correlations to develop on longer length scales, a partial re-initialization of the spin configuration would occur, unmasking the two different length scales characteristic of the two aging processest It is of interest to note that recent theoretical work 13 based on the droplet picture in a chaotic landscape can reproduce the experimentally observed rejuvenation and memory phenomena.

For comparison, The relaxation of the ZFC magnetization is investigated using the same cooling protocols as in the $M$ vs. $T$ measurements. Fig. 3 shows the relaxation curves obtained at $T_{m}=19 \mathrm{~K}$ (to compare with the single stop depicted in Fig. 1(a)). The different curves are measured in an applied field of 2 Oe according to the following protocols: 1, no stop was made during cooling to the lowest temperature and the magnetic field was applied $10 \mathrm{~s}$ after $T_{m}$ was reached on re-heating; $\mathbf{2}$, additionally to procedure 1 a stop was made for $3000 \mathrm{~s}$ at $T_{m}$ during cooling and $\mathbf{3}$, a conventional relaxation experiment at $T_{m}$ employing a wait time of $3000 \mathrm{~s}$ before applying the field. One notices that at observation times of $\sim 10-30$ s (corresponding to the effective observation time of the magnetization measurement on heating) and longer, curve $\mathbf{2}$ lies significantly below curve $\mathbf{1}$ as is also the case for the SSE magnetization compared to the no stop magnetization in Fig. 1(a)). It also shows that the SSE curve is almost indistinguishable from the curve recorded on a 3000s old system in a "conventional" ZFC relaxation measurement (curve $\mathbf{3}$ ). To illustrate the relative non-importance of the measurement field, the relaxation measurements illustrated in Fig. 3(a) are repeated, but cooling the system in the field $h_{i}=1$ Oe, and recording the magnetization with $h_{i}+2 \mathrm{Oe}=3$ Qe. The additional field cooled (FC) relaxation is small14, and as seen in Fig. $3(\mathrm{~b})$, the results are virtually unaffected by the presence of an initial field during the cooling and equilibration time. 
The DSE shown in Fig. 2 can also be mimicked by relaxation measurements, as illustrated in Fig. 1. Curve 1 and $\mathbf{2}($ resp $\mathbf{1 b}$ and $\mathbf{2 b})$ correspond to SSE at $(\mathrm{a}) T_{s}=T_{m}=21 \mathrm{~K}$ and (b) $T_{s}=T_{m}=17 \mathrm{~K}$. Curve $\mathbf{3}$ and $\mathbf{3} \mathbf{b}$ correspond instead to the DSE case, with stops at $T_{s 1}=21 \mathrm{~K}$ and $T_{s 2}=17 \mathrm{~K}$, measuring the relaxation at (a) $T_{m}=21 \mathrm{~K}$ or $(\mathrm{b}) T_{m}=17 \mathrm{~K}$. As indicated in the $M$ vs. $T$ measurements, the relaxation after the SSE and DSE protocols are virtually identical, illustrating again chaos and memory characteristics of the spin glass phase.

Note on the relaxation measurements. Looking again at Fig. 2, one notices that the relaxation 'dips' created in the $\Delta M$ vs. $T$ curves are rather broad, which makes it difficult to repeat DSE with closer stop temperatures. In that case, the relaxation experiments which are here used mainly to confirm the temperature dependent magnetization measurements, would yield more information on the interference and overlap effects 15 .

\section{CONCLUSION}

Using simple dc-magnetization measurements, performed after specific cooling protocols, memory and temperature chaos features are revealed in an Ising spin glass. The results are confirmed by relaxation measurements. It would be of interest to overcome the large 16 overlap observed in Monte Carlo simulations and reproduce these results by studying the correlation function at some temperature, after equilibration at a higher one.

\section{ACKNOWLEDGMENTS}

This work was financially supported by The Swedish Natural Science Research Council (NFR).

${ }^{1}$ D. S. Fisher and D. A. Huse, Phys. Rev B 38, 373 (1988); D. S. Fisher and D. A. Huse, Phys. Rev B 38, 386 (1988).

${ }^{2}$ A. J. Bray and M. A. Moore, Phys. Rev. Lett 58, 57 (1987).

${ }^{3}$ K. Jonason, E. Vincent, J. Hammann, J. P. Bouchaud and P. Nordblad, Phys. Rev. Lett. 81, 3243 (1998).

${ }^{4}$ T. Jonsson, K.Jonason, P. Jönsson and P. Nordblad, Phys. Rev. B 59, 8770 (1999).

${ }^{5}$ P. Granberg, L. Lundgren and P. Nordblad, J. Magn. Magn. Mater. 92, 228 (1990).

${ }^{6}$ R. Mathieu, P. Jönsson, D. N. H. Nam, and P. Nordblad, Phys. Rev. B 63, 92401 (2001).

7 J. Magnusson, C. Djurberg, P. Granberg, and P. Nordblad, Rev.Sci. Instrum. 68, 3761 (1997).

${ }^{8}$ A. Ito, H. Aruga, E. Torikai, M. Kikuchi, Y. Syono, and H. Takei, Phys. Rev. Lett. 57, 483 (1986).
${ }^{9}$ C. Djurberg, J. Mattsson, and P. Nordblad, Europhys. Lett. 29, 163 (1995).

${ }^{10}$ A. Ito, A. Tobo, N. Onchi, and J. Satooka, J. Phys. Soc. Jpn. 69, Suppl. A. pp. 223-227 (2000); L. W. Bernardi, H. Yoshino, K. Hukushima, H. Takayama, A. Tobo, and A. Ito, Phys. Rev. Lett. 86, 720 (2001).

${ }^{11}$ D. N. H. Nam, R. Mathieu, P. Nordblad, N. V. Khiem and N. X. Phuc, Phys. Rev. B 62, 8989 (2000).

12 Again, due to the chaotic nature of the SG phase, the equilibrium state at the lower temperature is distinct from the higher temperature one, so that the relaxation at the lower temperature is not affected by the first equilibration at the higher one.

${ }^{13} \mathrm{H}$. Yoshino, A. Lemaitre, J.-P. Bouchaud, condmat/0009152

14 T. Jonsson, K. Jonason and P. Nordblad, Phys. Rev. B 59, 9402 (1999).

${ }^{15}$ K. Jonason, P. Nordblad, E. Vincent, J. Hamman and J.-P. Bouchaud, Eur. Phys. J. B 13, 99 (2000); C. Djurberg, K. Jonason and P. Nordblad, Eur. Phys. J. B 10, 15 (1999)

${ }^{16}$ L. W. Bernardi, H. Takayama, private communications. 

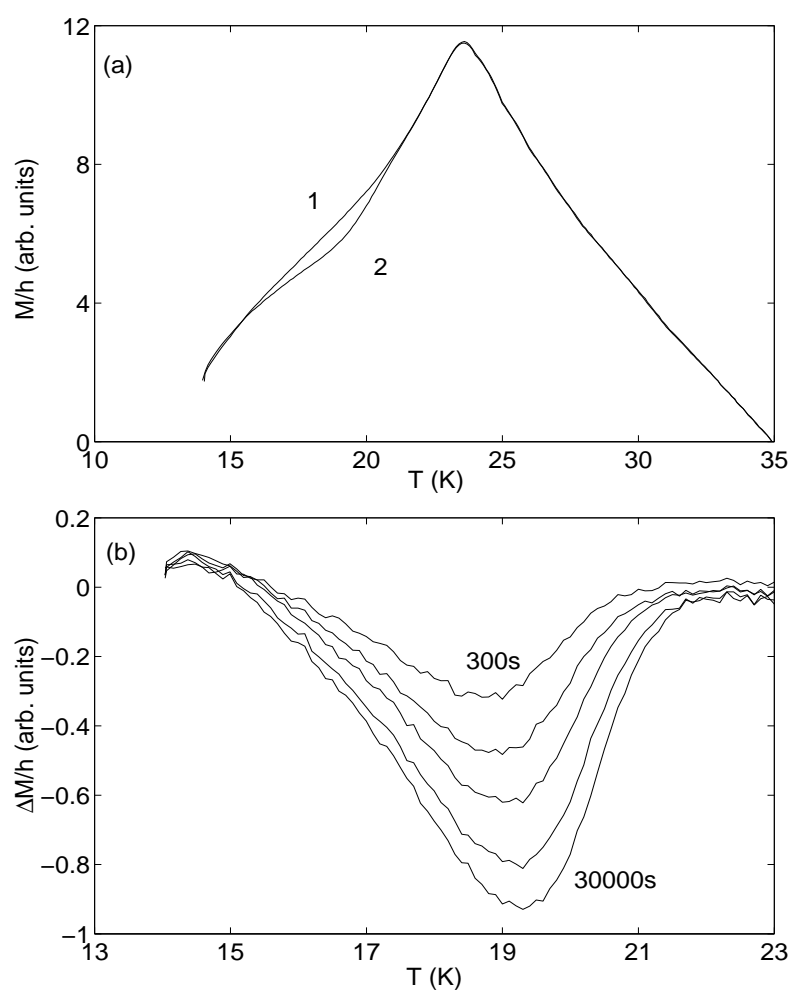

FIG. 1. (a) ZFC magnetization vs. temperature. Curve $\mathbf{1}$ is a reference curve, while curve $\mathbf{2}$ show the effects of a $3000 \mathrm{~s}$ stop at $T_{s}=19 \mathrm{~K}$ during the initial cooling. In (b) curve $\mathbf{2}$ is subtracted from the the reference; the results are shown for different waiting times, logarithmically spaced from 300 to 30000s

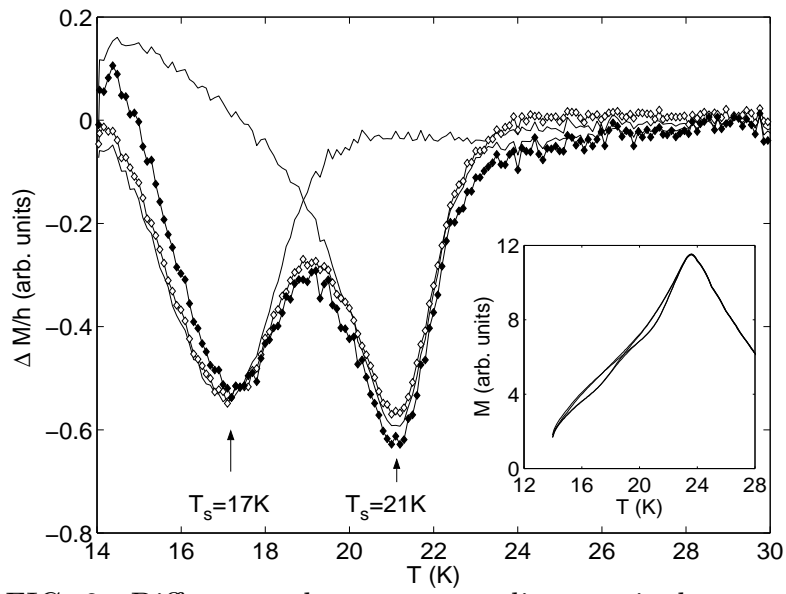

FIG. 2. Difference plots corresponding to single-stops of $3000 \mathrm{~s}$ at $T_{s}=17 \mathrm{~K}$ and $T_{s}=21 \mathrm{~K}$ (simple lines), and double-stops of $3000 \mathrm{~s}$ both at $T_{s 1}=21 \mathrm{~K}$ and $T_{s 2}=17 \mathrm{~K}$ (open diamonds). The sum of the two single-stop curves is added for comparison (filled diamonds). The insert shows the original ZFC magnetization vs. temperature curves.
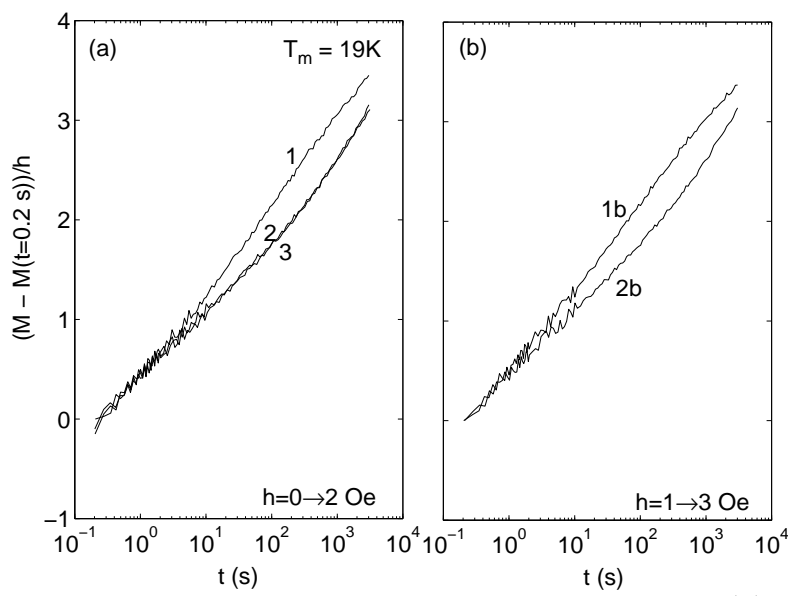

FIG. 3. ZFC relaxation curve for $\Delta h=2$ Oe. In (a) the sample is first cooled in zero magnetic field to the lowest temperature and re-heated to the measurement temperature $T_{m}=19 \mathrm{~K}$. After $t_{w}=10 \mathrm{~s}, \Delta h=2$ Oe is applied and the magnetization recorded vs. time. Curve $\mathbf{1}$ is a reference curve measured without a stop during cooling, while curve $\mathbf{2}$ shows the effects of an additional stop of $3000 \mathrm{~s}$ at $T_{s}=19 \mathrm{~K}$ during cooling. Curve $\mathbf{3}$ corresponds to a conventional ZFC relaxation experiment, with cooling down to the measurement temperature only, with $t_{w}=3000 \mathrm{~s}$. (b) shows the same experiments, performed this time cooling the sample is a field of $h=1$ Oe, and recording the magnetization in $h=3$ Oe.
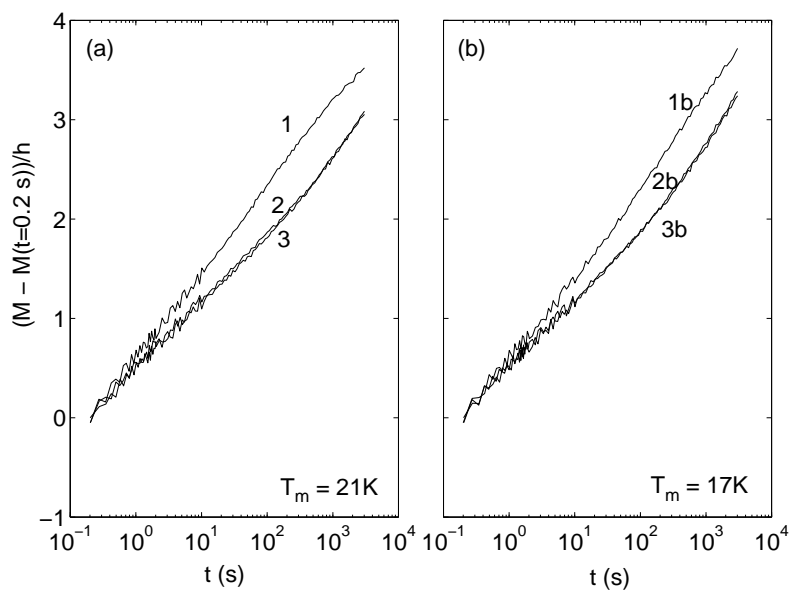

FIG. 4. Comparison to the double-stop experiments in $M$ vs. T. Curve $\mathbf{1}$ and $\mathbf{2}$ (resp. $\mathbf{1 b}$ and $\mathbf{2 b}$ ) correspond to the relaxation measurements described in Fig. 3(a), for (a) $T_{m}=21 \mathrm{~K}$ and (b) $T_{m}=17 \mathrm{~K}$. In the case of $\mathbf{3}$ (resp. $\mathbf{3 b}$ ), a first $3000 \mathrm{~s}$ stop in the initial cooling is made at $T_{s 1}=21 \mathrm{~K}$ before the second one at $T_{s 2}=17 \mathrm{~K}$. 